\title{
A Novel Technique for the Computation of Radiated EMI due to Corona on HV Transmission Lines
}

\author{
S.K. Nayak and M. Joy Thomas \\ Department of High Voltage Engineering \\ Indian Institute of Science, Bangalore, India-560012 \\ email: thoma@hve.iisc.ernet.in
}

\begin{abstract}
This paper presents a novel technique for the computation of radiated electromagnetic interference levels due to corona on $H V$ transmission lines. The EMI levels due to corona computed for different lines from around the world matches well with the experimentally measured values, thus validating the present technique. Using the technique developed, it has been shown that the radiated field at an observation point close to the transmission line increases with the line length and reaches almost a constant value for line lengths above 1500 $m$. The influence of increased spacing between phase conductors is to reduce the radiated field where as the type of phase configuration doesnot have any influence on the radiated field.
\end{abstract}

\section{Keywords}

Electromagnetic Interference, Corona, HV Transmission Lines

\section{INTRODUCTION}

Electromagnetic interference from high voltage transmission lines is caused by corona which is generated due to the electrical breakdown of the air surrounding the conductors at high voltage. When the conductor surface electric field exceeds the corona onset electric field, a partial breakdown occurs in the surrounding air near the conductor surface and is called the corona discharge.

The streamer generated during corona discharge transports electric charges into the surrounding air during a discharge cycle. These moving charges contribute directly to the noise fields. They also cause currents to be induced on the transmission line conductors. Since the charge is moved by a time varying electric field, it is equivalent to a current pulse and this current pulse is the source of time varying EMI field.

In the present work, an expression for radiated EMI field in time domain has been derived using Maxwell's equation. Using the expression thus derived, radiated electromagnetic fields due to corona on HV transmission lines have been computed. The computational results are in good agreement with the measured results reported in the literature [1].

\section{Electrical Characterstics of Corona Current Pulses}

The time varying corona current pulses generated during discharge cycle are double exponential in nature and can be

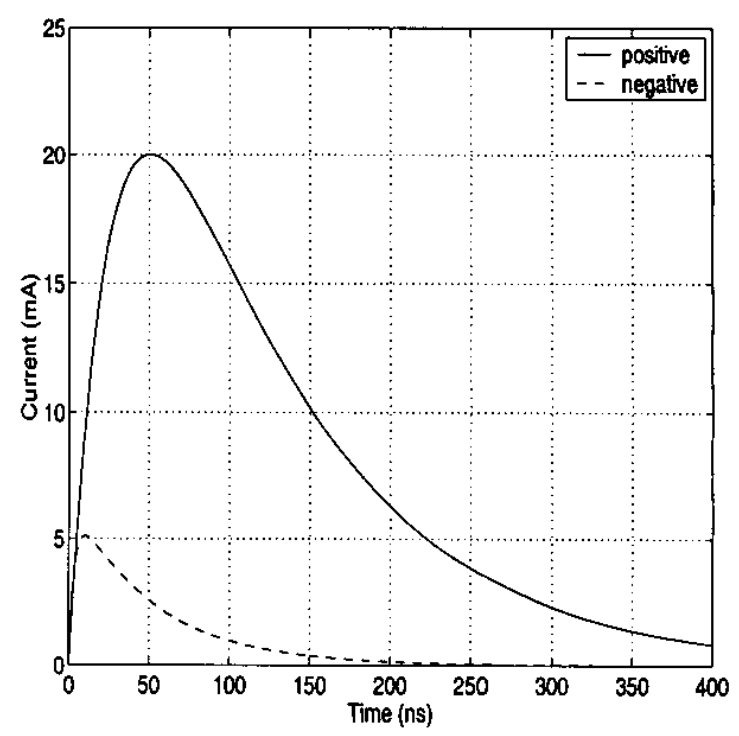

Fig. 1. Time domain representation of the corona current pulses represented by the following equation

$$
I(0, t)=K I_{p}\left(e^{-\alpha t}-e^{-\beta t}\right), t \geq 0
$$

Where $K, \alpha$ and $\beta$ are constants.

The time domain representation of the positive and negative corona current pulses are shown in figure 1.

Spatial Distribution of Coronating Points on Transmission Lines

Each corona discharge point radiates electric field and thus the total electric field at the observation point is due to the sum of the electric fields radiated from each corona discharge point. Since the corona phenomena is distributed along the transmission line, the length of the line certainly influences the magnitude of the total radiated EMI field at any observation point away from the line. It is observed that in fair weather there exits a certain shielding effect when one source in corona does not permit another within about 20-50 $\mathrm{cm}$ distance, ie., the distance between two adjacent corona discharge points lie in the range of $20-50 \mathrm{~cm}$ in fair weather conditions [2]. Also it has been observed that on an over head HV power line, positive corona pulses from a single 
point in corona occur once in a cycle or at the most 2 or 3 pulses are generated near the peak of the voltage waveform [2]. So for the computation, the entire transmission line length is divided into elementary segments each containing a coronating point and the radiated electric field for the total line at the observation point is the summation of electric field radiated from each of the segments.

\section{MATHEMATICAL FORMULATION OF THE RADI- ATED EM FIELD}

When the double exponential corona current pulse is injected and propagates along the conductor, electromagnetic waves are radiated by the conductor. The expression for the radiated electric field can be derived from Maxwell's equations by applying Lorentz Gauge condition and using Green's function. The derived expression for the radiated EMI field at the observation point ' $O$ ' is as follows [3]:

$$
\begin{aligned}
\vec{E}(P, t) & =-\frac{\mu_{0}}{8 \pi}\left[\frac{\widehat{s}}{R} \frac{\partial}{\partial \tau} I(s, \tau)+\frac{c \vec{R}}{R^{2}} \frac{\partial}{\partial s} I(s, \tau)\right. \\
+ & \frac{c^{2} \vec{R}}{R^{3}} \int_{0}^{\tau} \cdot \frac{\partial}{\partial s} I\left(s, t^{\prime}\right) d t^{\prime}+\frac{\widehat{s}_{m}}{R_{m}} \frac{\partial}{\partial \tau_{m}} I\left(s, \tau_{m}\right) \\
+ & \left.\frac{c \overrightarrow{R_{m}}}{R_{m}^{2}} \frac{\partial}{\partial s} I\left(s, \tau_{m}\right)-\frac{c^{2} \vec{R}_{m}}{R_{m}^{3}} \int_{0}^{\tau_{m}} \frac{\partial}{\partial s} I\left(s, t^{\prime}\right) d t^{\prime}\right]
\end{aligned}
$$

where $\tau=t-\frac{R}{c}$

$$
\tau_{m}=t-\frac{{ }^{c}}{c}
$$

$s$ is source point of the conductor

$s_{m}$ is the image point of the conductor

$R$ is the position vector of the conductor

$R_{m}$ is the position vector of the image conductor $c$ is velocity of light.

$I$ is double exponetial corona current pulse described earlier.

\section{Assumptions}

- The corona is uniformly distributed throughout the transmission line.

- The distance between two adjacent corona sources is 50 $\mathrm{cm}$.

- For the computational purpose, the maximum length of the transmission line has been taken as $1500 \mathrm{~m}$.

- Since '+ve' corona current pulses are of higher magnitude, the radiated field due to them only have been considered for the computation of maximum radiated EM field.

- The sag on the transmission line conductors has been neglected.

To compute the value of radiated electric field as given by equation (1), the length of the line is divided into a number of elementary segments ' $N$ seg' of length $50 \mathrm{~cm}$, since the distance between two adjacent corona pulses is assumed to be $50 \mathrm{~cm}$. Each $50 \mathrm{~cm}$ segment is further divided into a number of subsegments ' $N_{s}$ '.

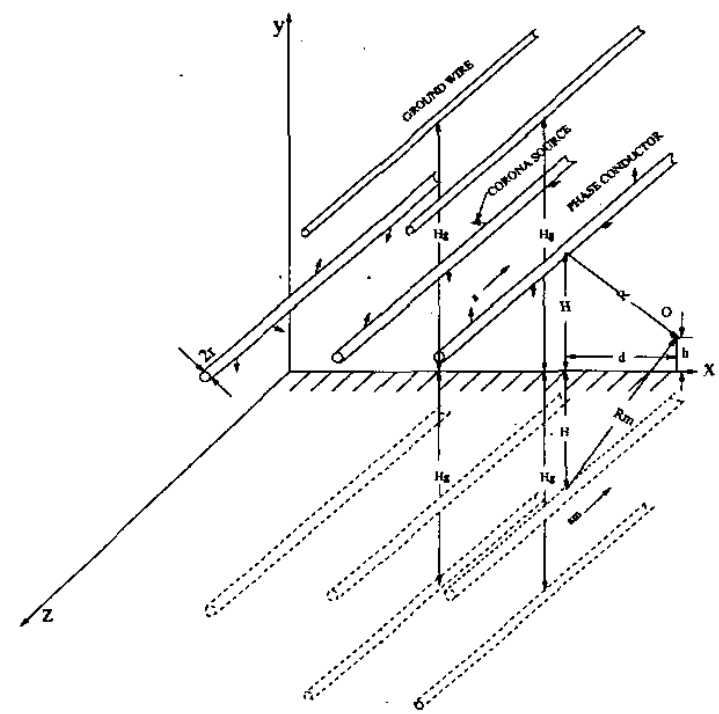

Fig. 2. Configuration of the transmission line used for computation

The space-time variation of radiated electric field at point ' $\mathrm{O}$ ' due to conductor corona and its image for an elementary length of the line $\Delta z$, is given as below.

$$
\begin{gathered}
\Delta \vec{E}(O, t)=-\frac{\mu_{0} \Delta z}{8 \pi}\left[\frac{\widehat{z}}{R} \frac{\partial}{\partial \tau} I(z, \tau)+\frac{c \vec{R}}{R^{2}} \frac{\partial}{\partial z} I(z, \tau)\right. \\
+\frac{c^{2} \vec{R}}{R^{3}} \int_{0}^{\tau} \frac{\partial}{\partial z} I\left(z, t^{\prime}\right) d t^{\prime}+\frac{\widehat{z}}{R_{m}} \frac{\partial}{\partial \tau_{m}} I\left(z, \tau_{m}\right) \\
\left.+\frac{c \overrightarrow{R_{m}}}{R_{m}^{2}} \frac{\partial}{\partial z} I\left(z, \tau_{m}\right)-\frac{c^{2} \overrightarrow{R_{m}}}{R_{m}^{3}} \int_{0}^{\tau_{m}} \frac{\partial}{\partial z} I\left(z, t^{\prime}\right) d t^{\prime}\right]
\end{gathered}
$$

where

$$
\begin{gathered}
\vec{R}=d \widehat{x}+(h-H) \widehat{y}+\left((n-1) \Delta z+\left(n_{s}-1\right) 0.5\right) \widehat{z} \\
\vec{R}_{m}=d \widehat{x}+(h+H) \widehat{y}+\left((n-1) \Delta z+\left(n_{s}-1\right) 0.5\right) \widehat{z} \\
I(z, t)=K I_{p}\left(e^{-\alpha t}-e^{-\beta t}\right) e^{-\alpha_{1} z}, t \geq 0 \\
n_{s}=123 \ldots N_{\text {seg. }}, \quad n=123 \ldots N_{s} .
\end{gathered}
$$

\section{Calculation of the Peak Value of Corona Current Pulse}

Let the line to ground voltage on the three phase conductors be $V_{1}=V_{p} \angle 0^{\circ}, V_{2}=V_{p} \angle-120^{\circ}$ and $V_{3}=V_{p} \angle+120^{\circ}$ respectively.

The voltage on the ground wire is taken to be zero.

The peak value of conductor surface electric field $E$ is given by [4]

$$
[E]=\frac{1}{2 \pi \epsilon_{o}} \frac{[Q]}{N r}\left[1+(N-1) \frac{r}{R}\right]
$$

where $[Q]=$ charge matrix.

$r=$ radius of subconductor.

$N=$ number of subconductors.

$R=$ bundle radius. 
The corona current, $J$, injected into conductor during corona discharge is given by

$$
[J]=e^{-\mu[E]^{2}}
$$

where $e^{-}=$charge of the electron

$$
\mu=\text { mobility of the electron }[4,5] \text {. }
$$

The peak value of corona current pulse propagating along the conductor is given by [6]

$$
I_{p}=\frac{[J]}{\sqrt{\alpha_{1}}}
$$

where $\alpha_{1}$ is the attenuation constant.

\section{RESULTS AND DISCUSSIONS}

For the computation of radiated electric field at the observation point, a $3 \phi$ transmission line as shown in figure 2 is taken for the study. The transmission line at a height of $\mathrm{H}$ (along the Y-axis) is assumed to be stressed with a voltage at power frequency. The ground wires are placed at a height of $H_{g}$. The effect of ground wires and image conductors on the radiated electric field are also taken into consideration. When the double exponential corona current pulse as shown in figure 1 is injected into the conductor during a discharge cycle, it radiates a field which is computed at the observation point ' $O$ ' which is at a height of $2 \mathrm{~m}$ from the ground. The effect of length and other parameters on the radiated electric field are discussed below.

\section{Comparision of Present Results with Measured Data}

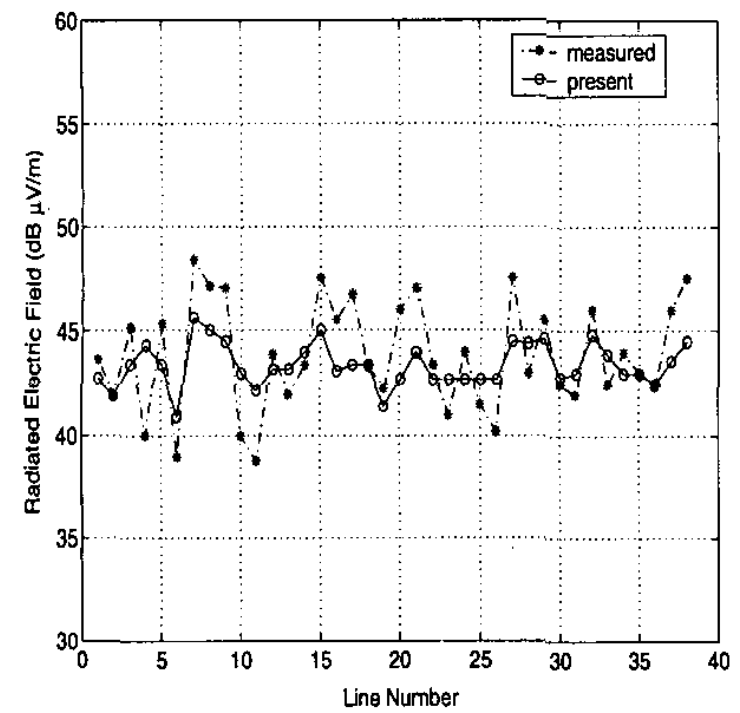

Fig. 3. Comparison of measured radiated field data with the calculated levels using the present technique.

\begin{tabular}{|c|c|c|}
\hline $\begin{array}{l}\text { Line } \\
\text { No }\end{array}$ & $\begin{array}{c}\text { Measured } \\
\text { radiated field } \\
\text { in } \mathrm{dB} \mu \mathrm{V} / \mathrm{m}\end{array}$ & $\begin{array}{c}\text { Computed } \\
\text { radiated field } \\
\text { in } \mathrm{dB} \mu \mathrm{V} / \mathrm{m}\end{array}$ \\
\hline$\overline{1}$ & 43.7 & $\overline{42.8}$ \\
\hline 2 & 42.0 & 41.9 \\
\hline 3 & 45.1 & 43.4 \\
\hline 4 & 40.0 & 44.3 \\
\hline 5 & 45.3 & 43.4 \\
\hline 6 & 39.0 & 40.9 \\
\hline 7 & 48.4 & 45.6 \\
\hline 8 & 47.1 & 45.0 \\
\hline 9 & 47.0 & 44.5 \\
\hline 10 & 40.0 & 43.0 \\
\hline 11 & 38.8 & 42.2 \\
\hline 12 & 43.9 & 43.2 \\
\hline 13 & 42.0 & 43.2 \\
\hline$\overline{14}$ & $\overline{43.4}$ & 44.0 \\
\hline 15 & 47.5 & 45.0 \\
\hline 16 & 45.5 & 43.1 \\
\hline 17 & 46.7 & 43.4 \\
\hline 18 & 43.3 & 43.4 \\
\hline 19 & 42.3 & 41.4 \\
\hline 20 & 46.0 & 42.7 \\
\hline 21 & 47.0 & 44.0 \\
\hline 22 & 43.4 & 42.7 \\
\hline 23 & 41.0 & 42.7 \\
\hline 24 & 44.0 & 42.7 \\
\hline 25 & 41.5 & 42.7 \\
\hline 26 & 40.2 & 42.7 \\
\hline 27 & 47.5 & 44.5 \\
\hline 28 & 43.0 & 44.3 \\
\hline 29 & 45.5 & 44.6 \\
\hline 30 & 42.5 & 42.8 \\
\hline 31 & 42.0 & 43.0 \\
\hline 32 & 46.0 & 44.8 \\
\hline 33 & 42.5 & 43.9 \\
\hline 34 & 44.0 & 43.0 \\
\hline 35 & 43.0 & 43.0 \\
\hline 36 & 42.4 & 42.5 \\
\hline 37 & 46.0 & 43.6 \\
\hline 38 & 47.5 & 44.5 \\
\hline
\end{tabular}

TABLE I

Comparison of measured and computed EMI values in fair weather

The EMI levels calculated using the method presented here are compared with the fair weather measured data of 38 different transmission lines from around the world. Table-1 summarises the radiated field data obtained from the survey [1] and by using the present method. The calculated levels along with the measured levels appear in figure 3 . The data are arranged in ascending order of the voltage level in $\mathrm{kV}$. As can be seen from the table, fair weather computed levels agree reasonably well with the measured data.

\section{Variation of Radiated Electromagnetic Field with Transmission Line Length}

The computed radiated electric field in time domain at the measurement point for different lengths of the transmission line are presented in figure 4 . The shape and pulse duration of the electric field remains the same as the length of the conductor increases but the peak value increases with the conductor length.

Figure 5 shows the variation in peak value of radiated electromagnetic field in $\mathrm{dB} \mu V / m$ with length of the line for the same measurement point. It can be observed that the field increases rapidly with increase of coronating conductor 


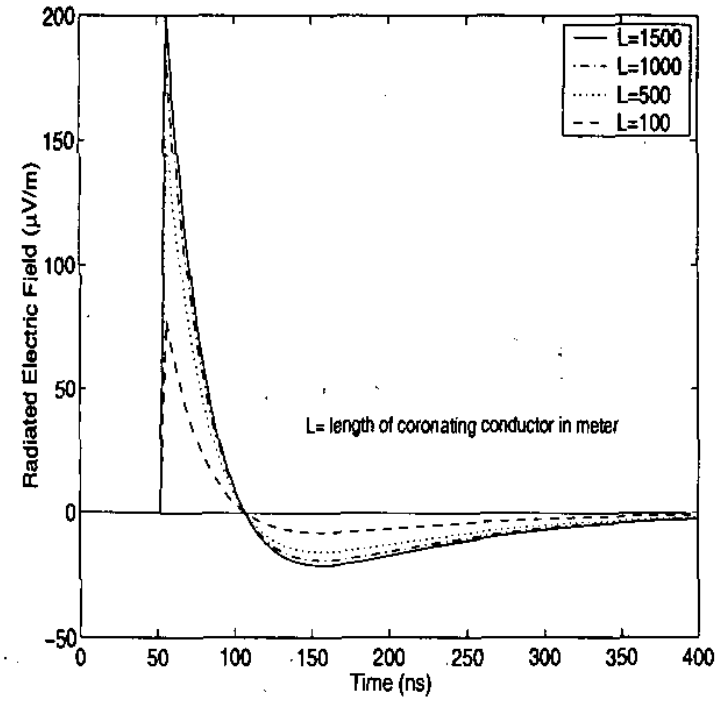

Fig. 4. Variation of radiated electric field in time domain for different coronating conductor length

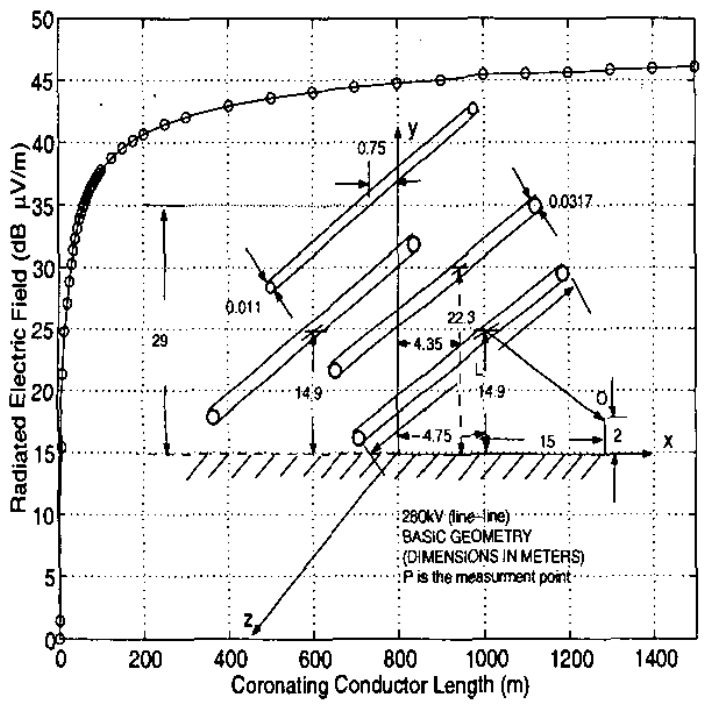

Fig. 5. Variation in peak value of the radiated EMI field with length of the coronating conductor

length upto $500 \mathrm{~m}$ and becomes almost constant for conductor lengths close to $1500 \mathrm{~m}$. The radiated electric field for the conductor length of $1500 \mathrm{~m}$ at the measurement point is $46.1 \mathrm{~dB}$ and for a length of $2000 \mathrm{~m}$ it is $46.6 \mathrm{~dB}$. Hence it may be suggested that for computation of EMI field due to corona at locations close to the transmission line corridor $(\simeq 15 \mathrm{~m})$, a line length of $750 \mathrm{~m}$ on either side of the observation point is good enough to get fairly accurate results.

\section{Variation of Radiated Electromagnetic Field with Measurement Distance}

The computed radiated electric field in time domain for the conductor length of $1000 \mathrm{~m}$ for the different measurement distances are presented in figure 6. The shape and the pulse duration of the radiated electric field remains the same as the measurement distance increases but reverse trend occurs in the peak value. When the distance of observation point increases, the propagation time $(\tau)$ of the electromagnetic field from the conductor to the measurement point increases.

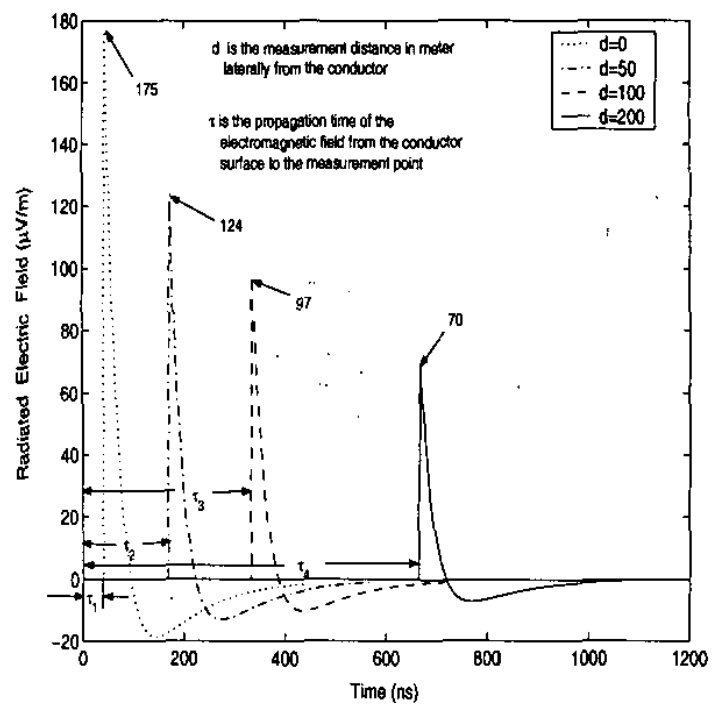

Fig. 6. Variation of radiated electric field in time domain for different measurement distances.

Figure 7 shows the variation of EMI levels for conductor heights of $15.2 \mathrm{~m}, 20.2 \mathrm{~m}$ and $25.2 \mathrm{~m}$. In general, as the height increases, the EMI level decreases. But after a certain lateral distance, the difference in EMI level for different heights show a reverse trend. This figure shows that there is not much variation in EMI levels with line height from the ground as the difference of height is less compared to the wavelength of the electromagnetic signal radiating from the conductor due to corona discharge. Figure 8 shows the EMI level variation for phase spacings of $5.7 \mathrm{~m}, 10.7 \mathrm{~m}$ and $15.7 \mathrm{~m}$ for different measurement distances laterally from the outer phase conductor, when the line is energized by a voltage of $528 \mathrm{kV}$ (line-line). The radiated electric field for the conductor length of $1000 \mathrm{~m}$ at measurement distance of $15 \mathrm{~m}$ are 45.2 $\mathrm{dB}, 43.2 \mathrm{~dB}$ and $41.6 \mathrm{~dB}$ for phase spacings of $5.7 \mathrm{~m}, 10.7 \mathrm{~m}$ and $15.7 \mathrm{~m}$ respectively. When the phase spacing increases, the influence of other phase conductors on the surface electric field decreases which leads to the decrease of EMI levels due to corona on transmission lines.

Variation of Radiated Electromagnetic Field with Conductor Length for Different Line Configurations In figure 9, the variation in EMI level with line length for three different line configurations has been studied. The radius of the transmission line conductor, measurement distance and height of the transmission lines are same for all the three configurations. When the transmission line conductor has been excited by voltage of $280 \mathrm{kV}$ (line-line), the EMI levels for 


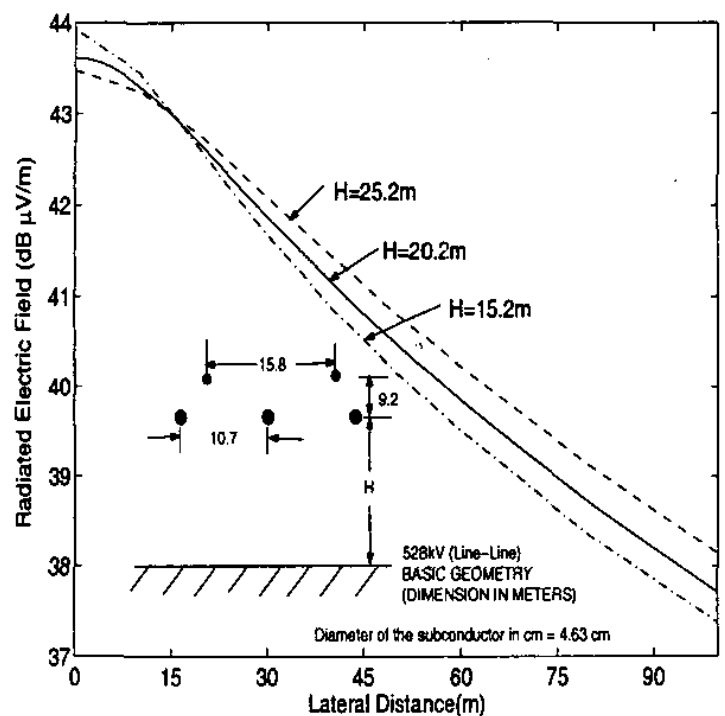

Fig. 7. Variation of radiated electric field with measurement distance for different conductor heights

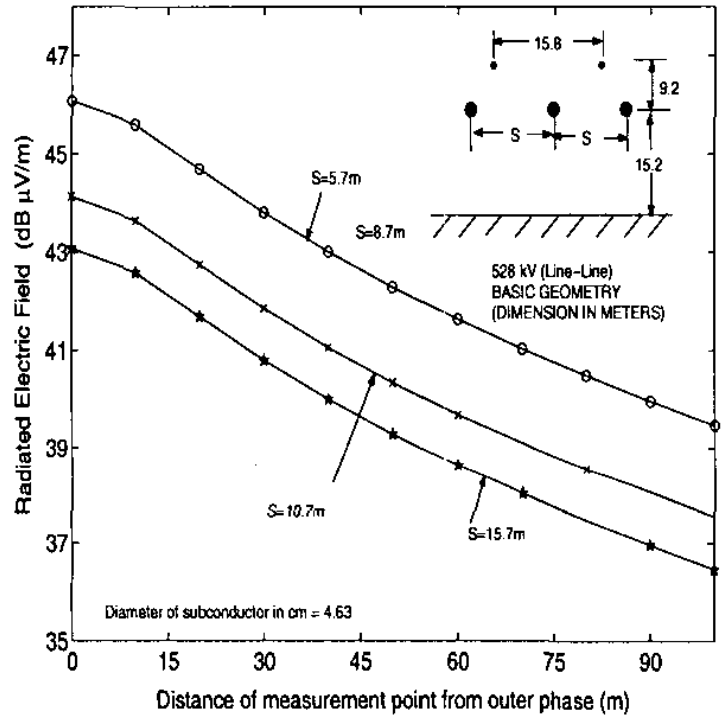

Fig. 8. Variation of radiated electric field with measurement distance for different phase spacings

a line length of $1500 \mathrm{~m}$ for the line configuration $\mathrm{A}, \mathrm{B}$ and $\mathrm{C}$ (shown in figure 9) are $44.4 \mathrm{~dB}, 46.1 \mathrm{~dB}$ and $45 \mathrm{~dB}$ respectively. The measurement point is at $15 \mathrm{~m}$ laterally from the outer phase conductor. Similar results have also been obtained by experimental measurements [1]. This indicates that the line configuration does not have much influence on the EMI level, due to the less variation of the conductor surface electric field for the three different configurations.

\section{CONCLUSIONS}

A novel technique for the computation of radiated electromagnetic interference levels due to corona on HV transmission lines has been developed based on field theoretical approach. The technique has been validated by comparing the

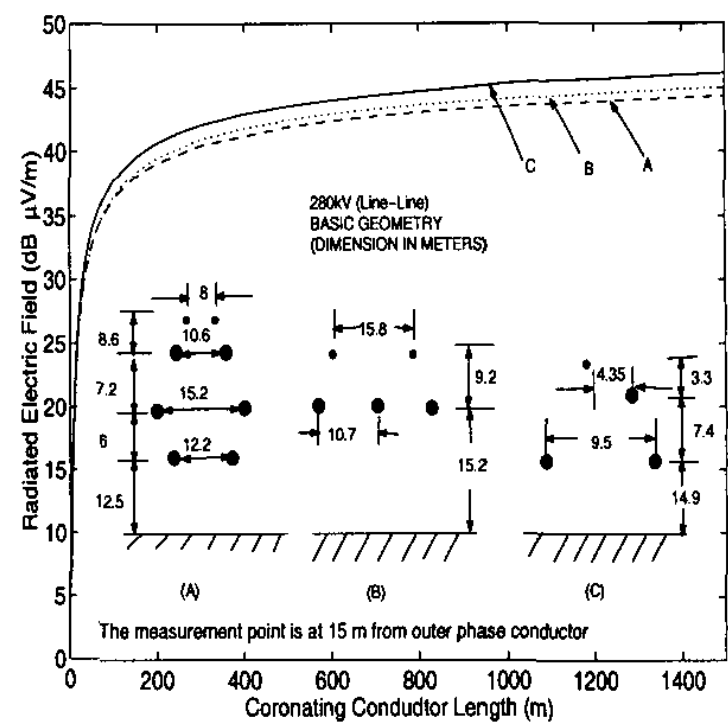

Fig. 9. Variation in radiated EMI field with length of the coronating conductor for different configurations of the transmission line

computed EMI levels of different lines from around the world with the experimentally measured values. It has been shown that the radiated field at an observation point close to the transmission line increases with the line length and reaches almost a constant value for line lengths above $1500 \mathrm{~m}$. The influence of increased spacing between phase conductors is to reduce the radiated field where as the type of transmission line phase configuration doesnot have any influence on the radiated field.

\section{REFERENCES}

[1] CIGRE/IEEE Survey on Extra High Voltage Transmission line Radio Noise, IEEE Transaction on Power Apparatus and Systems, Vol. PAS-92, No.3, pp.1019-1028, May/June 1973.

[2] Rokosh Das Begamudre, "Extra High Voltage A.C. Transmission Engineering", $2^{\text {nd }}$ Edition, New Age International (P) Limited, Publishers, New Delhi, p 95, 1990.

[3] C. BARDET, O. DAFIF, and B. JECKO, "Time Domain Analysis of a Large EMP Simulator", IEEE Transaction on EMC., Vol. EMC-29, No.1, pp. 40-48, Feb 1987.

[4] P. Sarma Maruvada, "Corona Performance of High Voltage Transmission Lines", Research Studies Press Ltd, Baldock, Hertfordshire, England, p 114-115, April 2000.

[5] D.K. Davies, "Measurements of swarm parameters in air", Theoretical Notes No. 346, Air Force Weapons Laboratory, USA, May 1983.

[6] M. R. Moreau and C. H. Gary, "Predetermination of the Radio-Interference Level of High Voltage Transmission Lines, Part II- Field Calculating Method." IEEE Transaction on Power Apparatus and Systems, Vol. PAS-91, No.1, pp.292-304, Jan/Feb 1972. 